\title{
Clostridium difficile infection: Antibodies versus antibiotics
}

\author{
Naddafi $F^{*}$ \\ Pharmaceutical Sciences Research Center, Shahid Beheshti University of Medical Sciences, Tehran, Iran
}

\begin{abstract}
Recently, the dramatic changes in the epidemiology of Clostridium difficile infection (CDI) as well as increases in both incidence and severity of disease in many countries have made it a global public health problem. This increasing severity and incidence could be partially because of frequent antibiotic use and the emergence of a hypervirulent strain of Clostridium difficile. Antibiotics such as metronidazole and vancomycin could be considered as frontline treatment for CDI. But recurrent CDI occurs in $\sim 25$ percent of cases and causes morbidity, mortality and healthcare costs. Due to antibiotic treatment failure in this population, novel treatment options are required. Recently, anti-toxin antibodies are developed as new therapeutic approach to the treatment of CDI. Bezlotoxumab, the first therapeutic monoclonal antibody, which is approved for the prevention of CDI recurrence. The aim of this manuscript is to provide the latest information about CDI treatment, with a particular focus on antibiotics and therapeutic antibodies that are utilized in CDI treatment.
\end{abstract}

\section{Introduction}

Clostridium difficile-associated disease (CDAD) which is caused by an anaerobic toxigenic bacterium could lead to significant morbidity and mortality worldwide [1,2]. Furthermore, it can cause a significant problem for solid organ transplant (SOT) recipients too [3]. Primary prevention of Clostridium difficile infection is considered as patients, without diarrhea symptoms, who receive antibiotics and are given the intervention within 2 months of treatment and do not develop diarrhea. Secondary prevention of CDI in cases who have recovered from at least one prior episode of CDI, without diarrhea at the time of the intervention and would not develop a recurrence of CDI within one or two months of follow-up [4]. Identification of both C. difficile toxin A (TcdA, enterotoxin) and toxin B (TcdB, cytotoxin) in diarrheal stool is required since these toxins are essential for C. difficile to induce both diarrhea and gut pathological changes $[5,6]$. It has been proved that tolevamer was inferior to antibiotic therapy for C. difficile infection (CDI) as well as metronidazole was inferior to vancomycin [7]. Both metronidazole and oral vancomycin have been clinically used for treatment of Clostridium difficile infection. However, clinical failure and concern about promotion of resistance motivated the search for new non-antibiotic treatments [8]. Furthermore, combination therapy (metronidazole and vancomycin) is not superior to monotherapy since it seems to be associated with an increase in the rate of adverse events [9]. In cases subgroups with C. difficile infection at increased recurrence risk, fidaxomicin, as first-line therapy, was cost-effective versus vancomycin. Moreover, it was less costly and more effective in cases with cancer [10]. Reduction in C. difficile environmental contamination by hospitalized cases that are treated with fidaxomicin was achieved [11]. In animal studies, the pooled relative risk of eight potential experiments indicated that the antibody therapy can reduce the risk of C. difficile infection [12]. Although preclinical studies confirmed the efficacy of actoxumab, bezolotoxumab alone was proved to be effective in clinical trials [13]. The antitoxin antibodies, actoxumab and bezlotoxumab, could bind to and neutralize TcdA and $\mathrm{TcdB}$, respectively. Moreover, bezlotoxumab was approved by the U.S. Food and Drug Administration in 2016 to reduce the recurrence of CDI in cases above 18 years of age who receives antibiotics for C. difficile infection and can be at a higher risk of recurrence [13,14]. Though only bezlotoxumab is shown to reduce recurrence of Clostridium difficile infection, studies using a combination of human monoclonal antibodies, actoxumab and bezlotoxumab, have indicated that bolstering the host immune response against both the C. difficile toxins might be effective in prevention of both primary and secondary C. difficile infection. But, since actoxumab development was halted, passive immunotherapy with both actoxumab and bezlotoxumab can be actually impracticable [15]. The superior effect of bezlotoxumab $(10 \mathrm{mg} / \mathrm{kg})$ in the prevention of recurrent CDI shows that the agent could be effective in Japanese patients [16]. Antibiotic therapy is augmented by using humanized monoclonal antibodies, actoxumab and bezlotoxumab, for the prevention of exotoxins A and B, respectively [14]. Bezlotoxumab, with a safety profile similar to that of placebo, had been associated with a substantially lower rate of recurrent infection than placebo (among cases who are receiving antibiotic treatment for primary / recurrent CDI). Furthermore, the addition of actoxumab could not improve efficacy [17]. It has been demonstrated that these monoclonal antibodies can be protective against CDI in hamsters [18] and piglets [19]. Moreover, they have shown promising results against recurrent CDI in humans $[20,21]$. It has been demonstrated that actoxumab-bezlotoxumab ]treatment can facilitate normalization of the gut microbiota in C. difficile infection mice [22]. A combination of these antibodies (referred to herein) is now in phase III clinical trials for the prevention of recurrent CDI. It has been shown that herein caused a 73 percent decrease in recurrence rates in phase II clinical trials When it was administered concurrently with the standard of care antibiotics such as vancomycin and metronidazole [20]. In another study, the use of chimeric fusion proteins is considered as an attractive approach to

${ }^{\star}$ Correspondence to: Fatemeh Naddafi, Pharmaceutical Sciences Research Center, Shahid Beheshti University of Medical Sciences, Tehran, Iran, E-mail: fatemeh.naddafi@yahoo.com

Key words: antibiotic treatment, bezlotoxumab, therapeutic monoclonal antibodies, recurrence, clostridium difficile infection

Received: March 25, 2019; Accepted: April 08, 2019; Published: April 11, 2019 
produce multivalent antitoxin vaccines as well as therapeutic polyclonal antibodies for both prevention and treatment of CDI [23].

\section{Conclusion}

C. difficile infections are considered as a leading cause of antibioticassociated diarrhea in both hospital and care facility cases. Antibiotics are known to target the infecting bacteria but not the exotoxins. Therefore, administering neutralizing antibodies against both TcdA and $\mathrm{TcdB}$ to cases who are receiving antibiotic therapy may modulate the effects of the exotoxins directly. Due to the limited number of drugs that have proven to be effective, treatment of CDI can be challenging followed by concerns about antibiotic resistance and recurring disease. More recent studies have provided additional insights into the potential value of utilizing monoclonal antibodies for treatment of CDI. Antibodies for the treatment of CDI have been confirmed to be effective in clinical practice and research. Monoclonal antibodies against $\mathrm{C}$. difficile toxins may protect against symptomatic CDI and subsequent CDI recurrences. A new approach for the prevention of recurrent CDI can be the use of therapeutic antibodies, as an adjunct to antibiotic therapy, directed against the toxins that are responsible for C. difficile infection. Bezlotoxumab is the first therapeutic monoclonal antibody for secondary prevention of recurrence of CDI. Though only bezlotoxumab is shown to reduce recurrence of CDI, previous data using a combination of actoxumab-bezlotoxumab have demonstrated that bolstering the host immune response against the C. difficile toxins could be effective in CDI prevention. Moreover, combination of two monoclonal antibodies might offer an advantage for a yet to emerge C. difficile strain, which can be a steady threat for cases at high risk of CDI. Furthermore, it has been shown that a combination therapy could facilitate normalization of the gut microbiota in CDI mice. Future studies will be required to assess human monoclonal antibodies combination as a therapeutic approach in both clinical and microbiological cure of Clostridium difficile infection.

\section{Conflicts of interest}

The author declared no conflicts of interest.

\section{References}

1. Burke KE, Lamont JT (2014) Clostridium difficile infection: A worldwide disease. Gut and liver $8:$ 1. [Crossref]

2. Balassiano I, Yates E, Domingues R, Ferreira E (2012) Clostridium difficile: a problem of concern in developed countries and still a mystery in Latin America. Journal of medical microbiology 61: 169-179. [Crossref]

3. Honda H, Dubberke ER (2014) Clostridium difficile infection in solid organ transplant recipients. Current opinion in infectious diseases 27: 336-341. [Crossref]

4. McFarland L (2015) Probiotics for the primary and secondary prevention of C. difficile infections: a meta-analysis and systematic review. Antibiotics 4: 160-178. [Crossref]

5. Ananthakrishnan AN (2011) Clostridium difficile infection: epidemiology, risk factors and management. Nature reviews Gastroenterology and hepatology 8: 17. [Crossref]

6. Qiu H, Cassan R, Johnstone D, Han X, Joyee AG, et al. (2016) Novel Clostridium difficile anti-toxin (TcdA and TcdB) humanized monoclonal antibodies demonstrate in vitro neutralization across a broad spectrum of clinical strains and in vivo potency in a hamster spore challenge model. PLoS One 11: e0157970. [Crossref]
7. Johnson S, Louie TJ, Gerding DN, Cornely OA, Chasan-Taber S, et al. (2014) Vancomycin, metronidazole, or tolevamer for Clostridium difficile infection: results from two multinational, randomized, controlled trials. Clinical Infectious Diseases 59: 345-354. [Crossref]

8. Rineh A, Kelso MJ, Vatansever F, Tegos GP, Hamblin MR, et al. (2014) Clostridium difficile infection: molecular pathogenesis and novel therapeutics. Expert review of anti-infective therapy 12: 131-150. [Crossref]

9. Li R, Lu L, Lin Y, Wang M, Liu X, et al. (2015) Efficacy and safety of metronidazole monotherapy versus vancomycin monotherapy or combination therapy in patients with Clostridium difficile infection: a systematic review and meta-analysis. PLoS One 10: e0137252. [Crossref]

10. Watt M, McCrea C, Johal S, Posnett J, Nazir J, et al. (2016) A cost-effectiveness and budget impact analysis of first line fidaxomicin for patients with Clostridium difficile infection (CDI) in Germany. Infection 44: 599-606.

11. Biswas J, Patel A, Otter J, Wade P, Newsholme W, et al. (2015) Reduction in Clostridium difficile environmental contamination by hospitalized patients treated with fidaxomicin. Journal of Hospital Infection 90: 267-270.

12. Diraviyam T, He J-X, Chen C, Zhao B, Michael A, et al. (2016) Effect of passive immunotherapy against Clostridium difficile infection: a systematic review and metaanalysis. Immunotherapy 8: 649-663. [Crossref]

13. Hernandez LD, Kroh HK, Hsieh E, Yang X, Beaumont M, et al. (2017) Epitopes and mechanism of action of the Clostridium difficile toxin A-neutralizing antibody actoxumab. Journal of molecular biology 429: 1030-1044. [Crossref]

14. Thakare R, Dasgupta A, Chopra S (2017) Bezlotoxumab for the treatment of Clostridium difficile-associated diarrhea. Drugs of today (Barcelona, Spain: 1998) 53: 385-92.

15. Posteraro B, Pea F, Masucci L, Posteraro P, Sanguinetti M (2018) Actoxumab+ bezlotoxumab combination: what promise for Clostridium difficile treatment? Expert opinion on biological therapy 18: 469-476.

16. Mikamo H, Aoyama N, Sawata M, Fujimoto G, Dorr MB, et al. (2018) The effect of bezlotoxumab for prevention of recurrent Clostridium difficile infection (CDI) in Japanese patients. Journal of Infection and Chemotherapy 24: 123-129. [Crossref]

17. Wilcox MH, Gerding DN, Poxton IR, Kelly C, Nathan R, et al. (2017) Bezlotoxumab for prevention of recurrent Clostridium difficile infection. New England Journal of Medicine 376: 305-317.

18. Babcock GJ, Broering TJ, Hernandez HJ, Mandell RB, Donahue K, et al. (2006) Human monoclonal antibodies directed against toxins A and B prevent Clostridium difficile-induced mortality in hamsters. Infection and immunity 74: 6339-6347.

19. Steele J, Mukherjee J, Parry N, Tzipori S (2012) Antibody against TcdB, but not TcdA prevents development of gastrointestinal and systemic Clostridium difficile disease. The Journal of infectious diseases 207: 323-330. [Crossref]

20. Lowy I, Molrine DC, Leav BA, Blair BM, Baxter R, Gerding DN, et al. (2010) Treatment with monoclonal antibodies against Clostridium difficile toxins. New England Journal of Medicine 362: 197-205. [Crossref]

21. Yang Z, Ramsey J, Hamza T, Zhang Y, Li S, Yfantis HG, et al. (2015) Mechanisms of protection against Clostridium difficile infection by the monoclonal antitoxin antibodies actoxumab and bezlotoxumab. Infection and immunity 83: 822-831. [Crossref]

22. Dzunkova M, D’Auria G, Xu H, Huang J, Duan Y, Moya A, et al. (2016) The monoclonal antitoxin antibodies (actoxumab-bezlotoxumab) treatment facilitates normalization of the gut microbiota of mice with Clostridium difficile infection. Frontiers in cellular and infection microbiology 6: 119. [Crossref]

23. Tian J-H, Glenn G, Flyer D, Zhou B, Liu Y, Sullivan E, et al. (2017) Clostridium difficile chimeric toxin receptor binding domain vaccine induced protection against different strains in active and passive challenge models. Vaccine 35: 4079-4087. [Crossref]

Copyright: (2019 Naddafi F. This is an open-access article distributed under the terms of the Creative Commons Attribution License, which permits unrestricted use, distribution, and reproduction in any medium, provided the original author and source are credited. 